\title{
Dirac equation in low dimensions: The factorization method
}

\author{
J. A. Sánchez-Monroy ${ }^{\mathrm{a}, *}$, C. J. Quimbay ${ }^{\mathrm{b}, \mathrm{c}, * *}$ \\ ${ }^{a}$ Instituto de Física, Universidade de São Paulo, 05508-090, São Paulo, SP, Brazil \\ ${ }^{b}$ Departamento de Física, Universidad Nacional de Colombia, Bogotá, D. C., Colombia \\ ${ }^{c}$ Associate researcher of CIF, Bogotá, Colombia
}

\begin{abstract}
We present a general approach to solve the $(1+1)$ and $(2+1)$-dimensional Dirac equations in the presence of static scalar, pseudoscalar and gauge potentials, for the case in which the potentials have the same functional form and thus the factorization method can be applied. We show that the presence of electric potentials in the Dirac equation leads to two Klein-Gordon equations including an energy-dependent potential. We then generalize the factorization method for the case of energy-dependent Hamiltonians. Additionally, the shape invariance is generalized for a specific class of energydependent Hamiltonians. We also present a condition for the absence of the Klein paradox (stability of the Dirac sea), showing how Dirac particles in low dimensions can be confined for a wide family of potentials.
\end{abstract}

Keywords: Klein paradox, Dirac sea stability, Supersymmetric quantum mechanics, Shape invariance, Energy-dependent Hamiltonian.

\footnotetext{
*Corresponding author

** Ciudad Universitaria, Building 404, Room 343, Bogotá, Colombia. Phone: (57)(1)3165000 Ext. 13051.

Email addresses: antosan@if.usp.br (J. A. Sánchez-Monroy), cjquimbayh@unal.edu.co (C. J. Quimbay) 


\section{Introduction}

The Dirac equation in the presence of different types of potentials leads to the prediction of some peculiar effects. Specifically, the presence of an electric scalar potential in the Dirac equation can imply the existence of the Klein paradox (instability of Dirac sea). However, in the context of elementary particle physics, this effect has not been experimentally observed until now due to the impossibility of having a large enough electric field. Thus, the effects produced by different kind of potentials in the Dirac equation can be studied by using quantum simulators $[1,2,3]$. For instance, Casanova et al. have shown how to engineer scalar, pseudoscalar and other linear potentials in the $(1+1)$-dimensional Dirac equation by manipulating two trapped ions [3]. Additionally, it is well known that the $(2+1)$-dimensional Dirac equation describes the low energy excitations of graphene [4]. In this sense, graphene might be considered as a simulator of quantum relativistic effects in $(2+1)$ dimensions. In particular, graphene allows a close realization of the Klein gedanken experiment [5] and this fact might be fundamental in the future design of graphene-based electronic devices.

Solutions of the Dirac equation in the presence of diverse types of potentials have been extensively addressed [6]-[22]. Among the different approaches to solve the Schrödinger and Dirac equations, we can find the factorization method, which is based on fundamental ideas proposed by Dirac [23] and Schrödinger [24]. This approach was subsequently generalized in a systematic way by Infeld et al. [25]. However, it is important to mention that the factorization method works in an equivalent way as the Darboux transformation does [26]. Additionally, the factorization method is analogous to the so called supersymmetric quantum mechanics (SUSYQM) proposed by Witten [27]. The SUSYQM has been applied to solve exactly problems in non-relativistic and relativistic quantum mechanics [8]. The SUSYQM technique allows to construct a pair of isospectral Hamiltonians (the so called supersymmetric partners) in terms of the ladder operators, which can be used to obtain the eigenvalues and eigenfunctions of the problem. In 1983, Gendenshtein [28] showed that if the supersymmetric partners are invariant under a discrete reparametrization (usually called shape invariance), the spectrum can be obtained algebraically.

The main goal of this work is to present a general approach to solve the $(1+1)$ and $(2+1)$-dimensional Dirac equations in the presence of static scalar, pseudoscalar and gauge potentials using the factorization method. We imple- 
ment this method for the case in which the potentials in the Dirac equation have the same functional form and only depend on one spatial cartesian coordinate. For the case in which the Dirac equation involves the presence of an electric scalar potential, we show that the two Klein-Gordon equations associated to the Dirac equation include an energy-dependent potential. Due to the fact that the factorization method has been only implemented, until now, for the case of energy-independent Hamiltonian, we generalize the factorization method for the case of energy-dependent Hamiltonians. Additionally, we generalize the shape invariance for a restricted class of energy-dependent Hamiltonians. Finally, we present a condition for the absence of the Klein paradox and therefore for the possible confinement of fermions. This condition is closely related to the existence of supersymmetric partners in the problem and this fact ensures that the Dirac sea is stable and does not mix the positive and negative energy states, as it was noted before by Martinez et al. in $(3+1)$-dimensions [29]. The hidden supersymmetry of the $(3+1)$ dimensional Dirac oscillator has been discussed by Benitez et al. [30] and the implication of supersymmetry on the stability of the Dirac sea was noted. Supersymmetry in the context of the Dirac equation has also been studied by other authors $[21,22]$.

The structure of this paper is the following: First, in section 1, we present a general method to solve the $(1+1)$-dimensional Dirac equation in the presence of static scalar, pseudoscalar and gauge potentials, for the case in which these potentials have the same functional form and thus the factorization method can be applied; in section 2, we first generalize the factorization method for the case of energy-dependent Hamiltonians, then we extend the shape invariance for a restricted class of energy-dependent Hamiltonians and finally we present some examples; in section 3, we show how this method can be applied to the case of the $(2+1)$-dimensional Dirac equation, but considering static external potentials depend only on one cartesian coordinate; in section 4, we present a condition for the absence of the Klein paradox and therefore for the possible confinement of fermions; finally in section 5, we present the conclusions of this work.

\section{The $(1+1)$-dimensional Dirac equation}

\subsection{Dirac equation in the presence of static potentials}

The $(1+1)$-dimensional Dirac equation in the presence of static scalar $V(x)$, pseudoscalar $P(x)$ and gauge $A_{\mu}(x)=\left\{A_{t}(x), A_{x}(x)\right\}$ potentials is 
written as

$$
\left[i \gamma^{\mu}\left(\hbar \partial_{\mu}+i \frac{e}{c} A_{\mu}(x)\right)-\frac{1}{c} P(x) \gamma^{5}-\frac{1}{c} V(x)-m c\right] \Psi(x, t)=0,
$$

where the Dirac matrices $\gamma^{\mu}$, with $\mu=0,1$, are the generators of the twodimensional flat space-time Clifford algebra given by

$$
\left\{\gamma^{\mu}, \gamma^{\nu}\right\}=2 \eta^{\mu \nu}
$$

and $\eta^{\mu \nu}=\operatorname{diag}(+,-)$. We choose the Dirac matrices to satisfy the following properties

$$
\left(\gamma^{0}\right)^{\dagger}=\gamma^{0} \text { and }\left(\gamma^{1}\right)^{\dagger}=-\gamma^{1}
$$

The Dirac equation (1) can be rewritten as follows $\left(\beta=\gamma^{0}, \alpha=\gamma^{0} \gamma^{1}\right)$

$$
i \hbar \frac{\partial}{\partial t} \Psi(x, t)=\mathcal{H} \Psi(x, t)=\left(c \alpha p+\beta m c^{2}+\mathcal{V}\right) \Psi(x, t)
$$

where

$$
\mathcal{V}=1 e A_{t}(x)-\alpha e A_{x}(x)+\beta V(x)+\beta \gamma^{5} P(x) .
$$

In the last expression, 1 stands for the $2 \times 2$ identity matrix and $\mathcal{H}$ is the Hamiltonian operator. Because there are only four linearly independent $2 \times 2$ matrices, then the potential matrix $\mathcal{V}$ is the most general combination of Lorentz structures $[12,13,14]$.

Let us consider the adjoint Hamiltonian $\left(\mathcal{H}^{\dagger}\right)$ given by

$$
\mathcal{H}^{\dagger}=\left(c \alpha p+\beta m c^{2}+\mathbf{1} e A_{t}(x)-\alpha e A_{x}(x)+\beta V(x)+\left(\gamma^{5}\right)^{\dagger} \beta(P(x))^{*}\right)
$$

where $^{*}$ denotes the complex conjugate. It was supposed that $A_{t}(x), A_{x}(x)$ and $V(x)$ are real-valued functions and we use the properties (3). Since $\mathcal{H}$ must be a Hermitian operator, there are two options: $\left(\gamma^{5}\right)^{\dagger}=-\gamma^{5}$ and $P(x)^{*}=P(x)$ or $\left(\gamma^{5}\right)^{\dagger}=\gamma^{5}$ and $P(x)^{*}=-P(x)$. In the next, we use the first option and then all the potentials are real functions. The $(1+1)$ dimensional stationary Dirac equation with a non-Hermitian and pseudoscalar interaction, has been examined $[31,32,33]$. In the next, we will focus on in Hermitian potentials.

In order to use an appropriate representation of the Dirac matrices $\gamma^{\nu}$, we choose

$$
\gamma^{0}=\sigma^{1}=\left(\begin{array}{ll}
0 & 1 \\
1 & 0
\end{array}\right), \quad \gamma^{1}=i \sigma^{3}=\left(\begin{array}{cc}
i & 0 \\
0 & -i
\end{array}\right)
$$




$$
\gamma^{5}=i \gamma^{0} \gamma^{1}=i \sigma^{2}=\left(\begin{array}{cc}
0 & 1 \\
-1 & 0
\end{array}\right)
$$

We can write the spinor $\Psi(x, t)$ as

$$
\Psi(x, t)=e^{-\frac{i E t}{\hbar}}\left(\begin{array}{l}
\psi_{1}(x) \\
\psi_{2}(x)
\end{array}\right)
$$

and then, from the Dirac equation (4), we obtain the following coupled equation system

$$
\begin{array}{r}
-c \hbar \frac{d}{d x}\left(\begin{array}{c}
\psi_{1}(x) \\
\psi_{2}(x)
\end{array}\right)+\left(\begin{array}{cc}
i e A_{x}(x)-V(x) & -e A_{t}(x)-P(x) \\
e A_{t}(x)-P(x) & i e A_{x}(x)+V(x)
\end{array}\right)\left(\begin{array}{l}
\psi_{1}(x) \\
\psi_{2}(x)
\end{array}\right) \\
=\left(\begin{array}{cc}
m c^{2} & -E \\
E & -m c^{2}
\end{array}\right)\left(\begin{array}{l}
\psi_{1}(x) \\
\psi_{2}(x)
\end{array}\right) .(10
\end{array}
$$

In the next, we will not consider the spacial component of the gauge potential $A_{x}(x)$ because the Coulomb gauge $(\nabla \cdot \vec{A}=0)$ can be used. For this reason, the condition $\frac{\partial A_{x}}{\partial x}=0$ is satisfied for the case of a $(1+1)$-dimensional static field. In general, this term can be canceled by means of a gauge transformation [14]. Thus, the problem of the Dirac equation in the presence of a gauge potential $A_{\mu}(x)$ can be studied consistently by considering only the presence of a static electric scalar potential $A_{t}(x)$ in the system.

\subsection{Implementation of the factorization method}

Now we implement the factorization method to obtain the spectrum associated to the Eq. (10). To do it, we restrict the treatment to the case of static potentials with the same functional form. In other words, these potentials only may differ from each other by a proportionality factor or a shift in their origin. For this reason, we assume that the potentials have the following form $V(x)=\zeta_{1} f(x)+\tilde{m} c^{2}, P(x)=\zeta_{2} f(x)+\varepsilon$ and $e A_{t}(x)=\zeta_{3} f(x)+\tilde{E}$, where $f(x)$ is a function on the variable $x$, and $\zeta_{i}, \varepsilon, \tilde{m} c^{2}$ and $\tilde{E}$ are real parameters. For this kind of potentials, the coupled equation system (10) can be written as

$$
\begin{aligned}
& -c \hbar \frac{d}{d x}\left(\begin{array}{c}
\psi_{1}(x) \\
\psi_{2}(x)
\end{array}\right)+V(x)\left(\begin{array}{cc}
-1 & -\ell-\zeta \\
\ell-\zeta & 1
\end{array}\right)\left(\begin{array}{l}
\psi_{1}(x) \\
\psi_{2}(x)
\end{array}\right) \\
& =\left(\begin{array}{cc}
(m+\tilde{m}) c^{2} & -(E-\tilde{E})+\varepsilon \\
(E-\tilde{E})+\varepsilon & -(m+\tilde{m}) c^{2}
\end{array}\right)\left(\begin{array}{l}
\psi_{1}(x) \\
\psi_{2}(x)
\end{array}\right),
\end{aligned}
$$


where $\zeta=\zeta_{2} / \zeta_{1}$ and $\ell=\zeta_{3} / \zeta_{1}$. We observe that if $\zeta=0$, then $\zeta_{2}=0$ and there is no presence of a pseudoscalar potential. Additionally, for $\ell=0$ we have $\zeta_{3}=0$, then for this case there is no presence of an electric scalar potential in the system. Without loss of generality, we set $\tilde{m}=\tilde{E}=0$, because $\tilde{m}$ and $\tilde{E}$ represent only a shift for the mass and energy terms. Now, for this kind of potentials, we can implement the factorization method. To implement this method, it is first necessary to diagonalize the matrix, which is multiplying $V(x)$, by multiplying it by a matrix $D$ from the left and by $D^{-1}$ from the right. The matrix $D$ is given by

$$
D=\frac{1}{2 \tau(1+\tau)}\left(\begin{array}{cc}
1+\tau & \zeta+\ell \\
\ell-\zeta & 1+\tau
\end{array}\right)
$$

where $\tau=\sqrt{1+\zeta^{2}-\ell^{2}}$. Introducing the notation

$$
D\left(\begin{array}{l}
\psi_{1}(x) \\
\psi_{2}(x)
\end{array}\right)=\left(\begin{array}{c}
\tilde{\psi}_{1}(x) \\
\tilde{\psi}_{2}(x)
\end{array}\right)
$$

the coupled equation system (11) can be written as

$$
\begin{aligned}
& -c \hbar \frac{d}{d x}\left(\begin{array}{c}
\tilde{\psi}_{1}(x) \\
\tilde{\psi}_{2}(x)
\end{array}\right)+V(x)\left(\begin{array}{cc}
-\tau & 0 \\
0 & \tau
\end{array}\right)\left(\begin{array}{c}
\tilde{\psi}_{1}(x) \\
\tilde{\psi}_{2}(x)
\end{array}\right) \\
& =\left(\begin{array}{cc}
\frac{E \ell+m c^{2}+\zeta \varepsilon}{\tau} & \frac{\varepsilon(1-\ell a+\tau)-a(1+\tau) m c^{2}-E(1+\zeta a+\tau)}{\tau(\tau+1)} \\
\frac{\varepsilon(1+\ell b+\tau)-b(1+\tau) m c^{2}+E(1+\zeta b+\tau)}{\tau(\tau+1)} & -\frac{E \ell+m c^{2}+\zeta \varepsilon}{\tau}
\end{array}\right)\left(\begin{array}{l}
\tilde{\psi}_{1}(x) \\
\tilde{\psi}_{2}(x)
\end{array}\right),
\end{aligned}
$$

where $a=\zeta+\ell$ and $b=\zeta-\ell$. The coupled equation system (14) can be rewritten in terms of the first-order differential operators $A$ and $A^{\dagger}$, which are known as ladder operators, in the following form

$$
\begin{aligned}
A \tilde{\psi}_{1}(x) & =w_{1} \tilde{\psi}_{2}(x), \\
A^{\dagger} \tilde{\psi}_{2}(x) & =w_{2} \tilde{\psi}_{1}(x),
\end{aligned}
$$

where the ladder operators are defined as

$$
\begin{aligned}
A & =c \hbar \frac{d}{d x}+\tau V(x)+\frac{E \ell+m c^{2}+\zeta \varepsilon}{\tau}, \\
A^{\dagger} & =-c \hbar \frac{d}{d x}+\tau V(x)+\frac{E \ell+m c^{2}+\zeta \varepsilon}{\tau},
\end{aligned}
$$


and the eigenvalues associated are

$$
\begin{aligned}
& w_{1}=\frac{a(1+\tau) m c^{2}-\varepsilon(1-\ell a+\tau)+E(1+\zeta a+\tau)}{\tau(\tau+1)}, \\
& w_{2}=-\frac{b(1+\tau) m c^{2}-\varepsilon(1+\ell b+\tau)-E(1+\zeta b+\tau)}{\tau(\tau+1)} .
\end{aligned}
$$

For the case in which $\tau \geq 0$, i.e. $1+\zeta^{2}>\ell^{2}$, the operators $A$ and $A^{\dagger}$ are mutually self-adjoint. On the other hand, for the case in which $\tau$ is purely imaginary, the operators $A$ and $A^{\dagger}$ are no longer mutually adjoint. In section 5, we will show that the last feature is related to the Klein paradox. The equation system (15) and (16) can be decoupled into the two following Klein-Gordon equations

$$
\begin{aligned}
& H_{-} \tilde{\psi}_{1}(x)=w \tilde{\psi}_{1}(x), \\
& H_{+} \tilde{\psi}_{2}(x)=w \tilde{\psi}_{2}(x),
\end{aligned}
$$

where the effective supersymmetric partners $H_{-}$and $H_{+}$are given in terms of the ladder operators as

$$
\begin{aligned}
& H_{-}=A^{\dagger} A, \\
& H_{+}=A A^{\dagger},
\end{aligned}
$$

and the eigenvalue $w$ is written as

$$
\begin{aligned}
& w=w_{1} w_{2} \\
& =\left(\frac{\left(E^{2}-m^{2} c^{4}\right) \zeta^{2}+\left(E+\ell m c^{2}\right)^{2}+\varepsilon^{2}\left(\ell^{2}-1\right)+2 \varepsilon\left(E \ell+m c^{2}\right) \zeta}{\tau^{2}}\right)
\end{aligned}
$$

From the definition of the ladder operators (17) and (18), we observe that the energy $E$ is multiplied by the parameter $\ell$. This means that the supersymmetric partners $H_{-}$and $H_{+}$are energy dependent. This fact shows that the presence of electric scalar potentials in the Dirac equation, i.e. $\ell \neq 0$, implies that the potentials in the two Klein-Gordon equations (21) and (22) depend on energy.

For the case $\zeta_{1}=\zeta_{3}=0$, we have the presence of a potential purely pseudoscalar in the problem. For this case, it is necessary to define the matrix $D$ consistently

$$
D=\left(\begin{array}{cc}
1 & 1 \\
-1 & 1
\end{array}\right) \text {. }
$$


Following a similar procedure as the one developed above, we obtain for this case the following equations

$$
\begin{aligned}
& A_{p} \tilde{\psi}_{1}(x)=w_{1} \tilde{\psi}_{2}(x), \\
& A_{p}^{\dagger} \tilde{\psi}_{2}(x)=w_{2} \tilde{\psi}_{1}(x),
\end{aligned}
$$

where the eigenvalues are given by $w_{1}=E+m c^{2}, w_{2}=E-m c^{2}$ and the ladder operators are

$$
\begin{gathered}
A_{p}=c \hbar \frac{d}{d x}+P(x), \\
A_{p}^{\dagger}=-c \hbar \frac{d}{d x}+P(x) .
\end{gathered}
$$

For this case, we observe directly from (27) and (28) that the associated Klein-Gordon equations are not including an energy-dependent potential.

\section{Factorization method for energy-dependent Hamiltonians}

In the previous section, we have shown how an energy-dependent Hamiltonian associated to the Dirac equation in the presence of static potentials has been factorized in terms of the ladder operators. However, until now, in the literature the factorization method has been only considered for energyindependent Hamiltonians. For this reason, in this section, we will show that this method can be consistently generalized for the case of energy-dependent Hamiltonians. To do it, we will follow an analogous procedure to the one presented by Dong [34] and Bagchi [35]. Here, it is important to mention that several studies about Hamiltonians including energy-dependent potentials can be found in the literature $[36,37,38,39]$. To start, we consider two differential operators $A(E)$ and $A^{\dagger}(E)$ mutually self-adjoint defined by

$$
\begin{gathered}
A(E)=c \hbar \frac{d}{d x}+W(x, E), \\
A^{\dagger}(E)=-c \hbar \frac{d}{d x}+W(x, E),
\end{gathered}
$$

where the real function $W(x, E)$ is generally known as the superpotential. With the help of this superpotential, the supersymmetric partners are written as

$$
\begin{gathered}
H_{-}(E)=A^{\dagger}(E) A(E), \\
H_{+}(E)=A(E) A^{\dagger}(E) .
\end{gathered}
$$


The discrete spectrum of $H_{ \pm}(E)$ associated to the eigenstates $\left\{\psi_{n}^{ \pm}\right\}$is respectively denoted as $\left\{E_{n}^{ \pm}\right\}$, with $n=0,1, \ldots$. We restrict now our interest to the case of unbroken supersymmetry systems, this means that the ground state eigenvalue of one of these Hamiltonians is zero. For this kind of systems, there are two different cases:

(i) If $A_{0} \tilde{\psi}_{0}^{-}=0$, therefore

$$
H_{-}\left(E_{0}^{-}\right) \tilde{\psi}_{0}^{-}=A^{\dagger}\left(E_{0}^{-}\right) A\left(E_{0}^{-}\right) \tilde{\psi}_{0}^{-}=0,
$$

then $\tilde{\psi}_{0}^{-}$is an eigenstate of $H_{-}$with a zero eigenvalue. For $n \geq 0$, the relation

$$
H_{-}\left(E_{n}^{-}\right) \tilde{\psi}_{n}^{-}=A^{\dagger}\left(E_{n}^{-}\right) A\left(E_{n}^{-}\right) \tilde{\psi}_{n}^{-}=E_{n}^{-} \tilde{\psi}_{n}^{-},
$$

implies

$$
H_{+}\left(E_{n}^{-}\right)\left(A\left(E_{n}^{-}\right) \tilde{\psi}_{n}^{-}\right)=A\left(E_{n}^{-}\right) A^{\dagger}\left(E_{n}^{-}\right) A\left(E_{n}^{-}\right) \tilde{\psi}_{n}^{-}=E_{n}^{-}\left(A\left(E_{n}^{-}\right) \tilde{\psi}_{n}^{-}\right) .
$$

Thus, for every eigenstate $\tilde{\psi}_{n}^{-}$of $H_{-}$, the function $A\left(E_{n}^{-}\right) \tilde{\psi}_{n}^{-}$is an eigenstate of $H_{+}$and its corresponding eigenvalue is $E_{n}^{-}$. Similarly, we have

$$
H_{+}\left(E_{n}^{+}\right) \tilde{\psi}_{n}^{+}=A\left(E_{n}^{+}\right) A^{\dagger}\left(E_{n}^{+}\right) \tilde{\psi}_{n}^{+}=E_{n}^{+} \tilde{\psi}_{n}^{+},
$$

therefore

$$
H_{-}\left(E_{n}^{+}\right)\left(A^{\dagger}\left(E_{n}^{+}\right) \tilde{\psi}_{n}^{+}\right)=A^{\dagger}\left(E_{n}^{+}\right) A\left(E_{n}^{+}\right) A^{\dagger}\left(E_{n}^{+}\right) \tilde{\psi}_{n}^{+}=E_{n}^{+}\left(A^{\dagger}\left(E_{n}^{+}\right) \tilde{\psi}_{n}^{-}\right) .
$$

Thus, for every eigenstate $\tilde{\psi}_{n}^{+}$of $H_{+}$, the function $A\left(E_{n}^{+}\right) \tilde{\psi}_{n}^{+}$is an eigenstate of $H_{-}$and its corresponding eigenvalue is $E_{n}^{+}$. Consequently, the following relations are obtained

$$
\begin{aligned}
& E_{n}^{+}=E_{n+1}^{-}, \quad E_{0}^{-}=0, \\
& \tilde{\psi}_{n}^{+}(x)=N_{n}^{+} A\left(E_{n+1}^{-}\right) \tilde{\psi}_{n+1}^{-}(x), n=0,1,2 \ldots, \\
& \tilde{\psi}_{n+1}^{-}(x)=N_{n+1}^{-} A^{\dagger}\left(E_{n}^{+}\right) \tilde{\psi}_{n}^{+}(x), n=1,2 \ldots,
\end{aligned}
$$

where $N_{n}^{+}$and $N_{n}^{-}$are normalization constants.

In order to ensure the conservation of the norm, for the case of energydependent potentials, it has been established that the probability density should be modified [36]. If the Hamiltonian for a $n$-eigenstate satisfies the equation $H_{n} \tilde{\psi}_{n}(x)=-\frac{\hbar^{2}}{2 m} \frac{\partial^{2} \tilde{\psi}_{n}(x)}{\partial x^{2}}+V\left(x, E_{n}\right) \tilde{\psi}_{n}(x)$, the wave functions are normalized if the condition

$$
\int d x\left|\tilde{\psi}_{n}(x)\right|^{2}\left[1-\frac{\partial V\left(x, E_{n}\right)}{\partial E_{n}}\right]=1
$$


is satisfied. For the problem considered here, it is not necessary to modify the probability density because the probability is conserved by means of the usual normalization condition

$$
\int d x\left(\left|\psi_{1}(x)\right|^{2}+\left|\psi_{2}(x)\right|^{2}\right)=1,
$$

where $\psi_{j}(x)=D^{-1} \tilde{\psi}_{j}(x)$, with $j=1,2$. For the case of supersymmetric partners, the problem of the dependence on the energy is entirely due to the method of diagonalization that is being used. The conservation of probability is ensured because the external potentials initially considered in the problem are independent of the energy.

(ii) If $A^{\dagger}\left(E_{0}^{+}\right) \tilde{\psi}_{0}^{+}=0$, then $\tilde{\psi}_{0}^{+}$is an eigenstate of $H_{+}$with an eigenvalue zero. For this case, we obtain the following relations

$$
\begin{aligned}
& E_{n}^{-}=E_{n+1}^{+}, \quad E_{0}^{+}=0, \\
& \tilde{\psi}_{n}^{-}(x)=N_{n}^{-} A^{\dagger}\left(E_{n+1}^{+}\right) \tilde{\psi}_{n+1}^{+}(x), n=0,1,2 \ldots, \\
& \tilde{\psi}_{n+1}^{+}(x)=N_{n+1}^{+} A\left(E_{n}^{-}\right) \tilde{\psi}_{n}^{-}(x), n=1,2 \ldots
\end{aligned}
$$

\subsection{Generalization of the shape invariance for energy-dependent Hamiltoni- ans}

As it was mentioned in the introduction, it is possible to obtain the spectrum algebraically for the case in which the pair of supersymmetric partners is isospectral and also shape invariant. But until now, the shape invariance has been studied in the literature only for energy-independent Hamiltonians. By this reason, we generalize the shape invariance for a specific class of energy-dependent Hamiltonians.

Since the supersymmetric partners $H_{+}$and $H_{-}$are products of the operator $A$ and its adjoint $A^{+}$, their eigenvalues are either zero or positive [40]. If these Hamiltonians have the same dependence on the variable and differ only on other parameters, i.e. these are invariant under a discrete reparametrization (shape invariance), we can obtain the energy spectrum as well as the eigenfunctions analytically.

For the case in which the ground state of $H_{-}$has associated a zero eigenvalue (case (i) of the previous subsection), the shape invariance condition is given by

$$
H_{+}\left(a_{1}, x, E\right)=H_{-}\left(a_{2}, x, E\right)+R\left(a_{1}, E\right),
$$


where $a_{s}=f^{s-1}\left(a_{1}\right)$ (i.e., $f$ is applied $s-1$ times). For the particular case in which $R\left(a_{s}\right)$ is not dependent on $E$, we have

$$
\begin{aligned}
E_{1}^{-}\left(a_{1}\right) & =E_{0}^{+}\left(a_{1}\right)=E_{0}^{-}\left(a_{2}\right)+R\left(a_{1}\right) \\
& =R\left(a_{1}\right)
\end{aligned}
$$

and

$$
\begin{aligned}
E_{2}^{-}\left(a_{1}\right) & =E_{1}^{+}\left(a_{1}\right)=E_{1}^{-}\left(a_{2}\right)+R\left(a_{1}\right) \\
& =E_{0}^{+}\left(a_{2}\right)+R\left(a_{1}\right) \\
& =E_{0}^{-}\left(a_{3}\right)+R\left(a_{2}\right)+R\left(a_{1}\right) \\
& =R\left(a_{2}\right)+R\left(a_{1}\right) .
\end{aligned}
$$

In general, the spectrum is given by

$$
E_{n}^{-}=\sum_{k=1}^{n} R\left(a_{k}\right),
$$

and the eigenfunction describing the ground state can be written as

$$
\tilde{\psi}_{0}^{-}\left(x, a_{1}\right)=N_{0}^{-} \exp \left(-\int^{x} \frac{W\left(x, E_{0}^{-}, a_{1}\right)}{c \hbar} d x\right) .
$$

From the shape invariant condition Eq. (48), we have that $H_{+}\left(a_{1}, x, E\right)$ and $H_{-}\left(a_{2}, x, E\right)$ have the same eigenfunctions. The eigenfunction describing the ground state satisfies

$$
\tilde{\psi}_{0}^{+}\left(x, a_{1}\right)=\tilde{\psi}_{0}^{-}\left(x, a_{2}\right) \sim \exp \left(-\int^{x} \frac{W\left(x, E_{0}^{-}, a_{2}\right)}{c \hbar} d x\right),
$$

therefore

$$
\tilde{\psi}_{1}^{-}\left(x, a_{1}\right) \sim A^{\dagger}\left(E_{0}^{+}, a_{1}\right) \tilde{\psi}_{0}^{+}\left(x, a_{1}\right)=A^{\dagger}\left(E_{1}^{-}, a_{1}\right) \tilde{\psi}_{0}^{-}\left(x, a_{2}\right)
$$

and

$$
\begin{aligned}
\tilde{\psi}_{2}^{-}\left(x, a_{1}\right) & \sim A^{\dagger}\left(E_{1}^{+}, a_{1}\right) \tilde{\psi}_{1}^{+}\left(x, a_{1}\right)=A^{\dagger}\left(E_{2}^{-}, a_{1}\right) \tilde{\psi}_{1}^{-}\left(x, a_{2}\right) \\
& =A^{\dagger}\left(E_{2}^{-}, a_{1}\right) A^{\dagger}\left(E_{1}^{-}, a_{2}\right) \tilde{\psi}_{0}^{-}\left(x, a_{3}\right) .
\end{aligned}
$$

For $n>0$, in general, we have

$$
\tilde{\psi}_{n}^{-}\left(x, a_{1}\right) \sim A^{\dagger}\left(E_{n}^{-}, a_{1}\right) \ldots A^{\dagger}\left(E_{2}^{-}, a_{n-1}\right) A^{\dagger}\left(E_{1}^{-}, a_{n}\right) \tilde{\psi}_{0}^{-}\left(x, a_{n+1}\right) .
$$




\subsection{Examples}

We now consider two examples in which the spectrum of an energydependent Hamiltonian is calculated using the generalizations of the factorization method and shape invariance previously presented.

\subsubsection{Linear potential}

Let us consider the system defined by the $(1+1)$-dimensional Dirac equation in the presence of a linear potential of the form $f(x)=\frac{a x}{\tau \zeta_{1}}$. For this system, the ladder operators are

$$
\begin{aligned}
A & =c \hbar \frac{d}{d x}+a x+b_{n}, \\
A^{\dagger} & =-c \hbar \frac{d}{d x}+a x+b_{n},
\end{aligned}
$$

where $b_{n}=\left(E_{n} \ell+m c^{2}+\zeta \varepsilon\right) / \tau$. The supersymmetric partners are

$$
\begin{aligned}
& H_{-}=-(c \hbar)^{2} \frac{d^{2}}{d x^{2}}+a^{2} x^{2}+2 a b_{n} x+b_{n}^{2}-c \hbar a, \\
& H_{+}=-(c \hbar)^{2} \frac{d^{2}}{d x^{2}}+a^{2} x^{2}+2 a b_{n} x+b_{n}^{2}+c \hbar a .
\end{aligned}
$$

These Hamiltonians are shape invariants because the condition (48) is satisfied

$$
H_{+}(a, x)=H_{-}(a, x)+2 c \hbar a .
$$

For this system $a_{s}=a, R\left(a_{s}\right)=2 c \hbar a$ and the eigenvalues of $H_{-}$are

$$
w_{n}^{-}=\sum_{s=1}^{n} R\left(a_{s}\right)=\sum_{s=1}^{n} 2 c \hbar a=2 c \hbar a n .
$$

For the case $a>0$, the quantity $w_{0}$ corresponds to the ground state eigenvalue and the condition $w_{0}<w_{1}<w_{2}<\ldots$ is satisfied. For the case $a<0$, we can obtain from (61) the condition

$$
H_{-}(a, x)=H_{+}(a, x)-2 c \hbar a,
$$

which implies that the eigenvalues of $H_{+}$are

$$
w_{n}^{+}=\sum_{s=1}^{n} R\left(a_{s}\right)=-\sum_{s=1}^{n} 2 c \hbar a .
$$


In this form, the eigenvalues of the $(1+1)$-dimensional Dirac equation in the presence of static scalar, pseudoscalar and electric scalar potentials are obtained from (25) and are given by

$$
E_{n}=\frac{-\ell\left(m c^{2}+\zeta \epsilon\right) \pm \sqrt{\tau^{2}\left(\left(\epsilon-m c^{2} \zeta\right)^{2}+2|a| c \hbar n\left(1+\zeta^{2}\right)\right)}}{1+\zeta^{2}} .
$$

This spectrum is symmetric for $\ell=0$, i.e. for a vanishing electric scalar potential $A_{t}=0$, and is only valid if the inequality $1+\zeta^{2}>\ell^{2}$ is satisfied. The spectrum of a Dirac particle in $(1+1)$ dimensions in the presence of a scalar field obtained by Long et al. [11] is reproduced here from (65), for the case $\zeta=\epsilon=\ell=0$.

\subsubsection{Inversely linear potential}

Now we consider the system defined by the $(1+1)$-dimensional Dirac equation in the presence of an inversely linear potential of the form $f(x)=$ $-\frac{q}{\zeta_{1} x}$, with $q>0$, where the variable $x$ is defined in the positive real line $\left(x \in \mathbb{R}^{+}\right)$. For this system, the ladder operators are

$$
\begin{aligned}
A & =c \hbar \frac{d}{d x}-\frac{\tilde{\tau}}{x}+f_{n}, \\
A^{\dagger} & =-c \hbar \frac{d}{d x}-\frac{q}{x}+f_{n},
\end{aligned}
$$

with $f_{n}=\left(E_{n} \ell+m c^{2}+\zeta \varepsilon\right) / \tau$ and $\tilde{\tau}=q \tau$. The supersymmetric partners are written as

$$
\begin{aligned}
& H_{-}=-(c \hbar)^{2} \frac{d^{2}}{d x^{2}}+\frac{\tilde{\tau}(\tilde{\tau}-c \hbar)}{x^{2}}-\frac{2 f_{n} \tilde{\tau}}{x}+f_{n}^{2}, \\
& H_{+}=-(c \hbar)^{2} \frac{d^{2}}{d x^{2}}+\frac{\tilde{\tau}(\tilde{\tau}+c \hbar)}{x^{2}}-\frac{2 f_{n} \tilde{\tau}}{x}+f_{n}^{2} .
\end{aligned}
$$

It is possible to show that these Hamiltonians are explicitly shape invariants if the change of variable $\rho=f_{n} \tilde{\tau} x$ is performed into the operators (66) and (67). After this change of variable, the operators (66) and (67) are written respectively as

$$
\begin{aligned}
\tilde{A} & =c \hbar \frac{d}{d \rho}-\frac{\tilde{\tau}}{\rho}+\frac{1}{\tilde{\tau}} \\
\tilde{A}^{\dagger} & =-c \hbar \frac{d}{d \rho}-\frac{\tilde{\tau}}{\rho}+\frac{1}{\tilde{\tau}}
\end{aligned}
$$


These operators are the same that those obtained when the $(3+1)$-dimensional problem is solved [8]. With the new operators (70) and (71), the following equations are satisfied

$$
\begin{aligned}
& \tilde{H}_{-} \tilde{\psi}^{-}(\rho)=\tilde{A}^{\dagger} \tilde{A} \tilde{\psi}^{-}(\rho)=\tilde{w} \tilde{\psi}^{-}(\rho)=\frac{w}{f_{n}^{2} \tilde{\tau}^{2}} \tilde{\psi}^{-}(\rho), \\
& \tilde{H}_{+} \tilde{\psi}^{+}(\rho)=\tilde{A} \tilde{A}^{\dagger} \tilde{\psi}^{+}(\rho)=\tilde{w} \tilde{\psi}^{+}(\rho)=\frac{w}{f_{n}^{2} \tilde{\tau}^{2}} \tilde{\psi}^{+}(\rho) .
\end{aligned}
$$

For this case, the shape invariance leads to

$$
\begin{gathered}
\tilde{H}_{+}(\tilde{\tau}, \rho)=\tilde{H}_{-}(\tilde{\tau}+c \hbar, \rho)+\frac{1}{\tilde{\tau}^{2}}-\frac{1}{(\tilde{\tau}+c \hbar)^{2}}, \\
a_{2}=\tilde{\tau}+c \hbar, \quad a_{1}=\tilde{\tau}, \quad R\left(a_{2}\right)=\frac{1}{\tilde{\tau}^{2}}-\frac{1}{(\tilde{\tau}+c \hbar)^{2}} .
\end{gathered}
$$

Thus, the eigenvalues for this case are

$$
\frac{w_{n}}{f_{n}^{2} \tilde{\tau}^{2}}=\sum_{j=1}^{n} R\left(a_{j}\right)=\frac{1}{\tilde{\tau}^{2}}-\frac{1}{(\tilde{\tau}+n c \hbar)^{2}}
$$

implying that the spectrum is given by the solutions of

$$
w_{n}=f_{n}^{2}\left(1-\frac{\tilde{\tau}^{2}}{(\tilde{\tau}+n c \hbar)^{2}}\right) .
$$

In general, the expression for the spectrum is complicated but we can obtain simple analytical expressions of this spectrum for two special cases:

(i) For $l=0$, we have the case of a mix between scalar and pseudoscalar potentials and there is no presence of an electric scalar potential. For this case, the spectrum is given by

$$
E_{n}= \pm \frac{\left(m c^{2}+\zeta \varepsilon\right)}{\tau} \sqrt{\frac{1}{\tau^{2}}-\frac{q^{2}}{(q \tau+n c \hbar)^{2}}+\frac{\left(m c^{2} \zeta-\varepsilon\right)^{2}}{\left(m c^{2}+\varepsilon \zeta\right)^{2}}}
$$

where $\tau=\sqrt{1+\zeta^{2}}$. If $\zeta=0$ and $\varepsilon=0$, the energy spectrum that we obtain from (78) corresponds to the one presented by Castro [15], for the case of a symmetric scalar potential of the form $V(x)=-\frac{k}{|x|}$. 
(ii) For $\zeta=0$ and $\varepsilon=0$, we have the case of a mix between scalar and electric scalar potentials. For this case, the spectrum is given by

$$
E_{n}=\frac{m c^{2}}{(q l)^{2}+(n c \hbar+\tilde{\tau})^{2}}\left(-q^{2} l \pm(n c \hbar+\tilde{\tau}) \sqrt{(n c \hbar+\tilde{\tau})^{2}-\tilde{\tau}^{2}}\right)
$$

where $\tilde{\tau}=q \sqrt{1-l^{2}}$. This result is in agreement with the spectrum found by Castro [16], who has shown that the problem can be mapped into a SturmLiouville problem in such a way that the spectrum can be obtained. For the case of two and three spacial dimensions, Xing et al. have solved the Dirac equation in the presence of scalar and electric scalar potentials [41], by using the same method that we have developed in this work.

\section{The $(2+1)$-dimensional Dirac equation}

The $(2+1)$-dimensional Dirac equation in the presence of scalar $V(x, y)$ and gauge $A_{\mu}(x, y)$ potentials is given by

$$
\left[i \gamma^{\mu}\left(\hbar \partial_{\mu}+i \frac{e}{c} A_{\mu}(x, y)\right)-\frac{1}{c} V(x, y)-m c\right] \Psi(\vec{r}, t)=0,
$$

where the Dirac matrices $\gamma^{\mu}$, with $\mu=0,1,2$, are the generators of the Clifford algebra in the three-dimensional flat spacetime

$$
\left\{\gamma^{\mu}, \gamma^{\nu}\right\}=2 \eta^{\mu \nu}
$$

There are two non-equivalent representations of the Dirac matrices in the $(2+1)$ dimensional case

$\gamma^{0}=\sigma^{3}=\left(\begin{array}{cc}1 & 0 \\ 0 & -1\end{array}\right), \quad \gamma^{1}=s i \sigma^{1}=s\left(\begin{array}{ll}0 & i \\ i & 0\end{array}\right), \quad \gamma^{2}=i \sigma^{2}=\left(\begin{array}{cc}0 & 1 \\ -1 & 0\end{array}\right)$

where the parameter $s= \pm 1$ characterizes the two possible values of the electron spin projection and $\sigma^{i}$ are the usual Pauli matrices. Taking into account that $\beta=\gamma^{0}=\sigma^{3}, \alpha_{1}=\gamma^{0} \gamma^{1}=-s \sigma^{2}$ and $\alpha_{2}=\gamma^{0} \gamma^{2}=\sigma^{1}$, the Dirac equation (80) can be rewritten as follows

$$
i \hbar \frac{\partial}{\partial t} \Psi(x, y, t)=\mathcal{H} \Psi(x, y, t)=c \alpha_{1} p_{x}+c \alpha_{2} p_{y}+\beta m c^{2}+\mathcal{V}
$$


where

$$
\mathcal{V}=I e A_{t}(x, y)+\sigma^{1} e A_{y}(x, y)-s \sigma^{2} e A_{x}(x, y)+\sigma^{3} V(x, y)
$$

with $I$ representing the $2 \times 2$ identity matrix. The potential matrix $\mathcal{V}$ corresponds to the most general combination of Lorentz structures because there are only four linearly independent $2 \times 2$ matrices [12]. If we write the spinor $\Psi(x, y, t)$ as

$$
\Psi(x, y, t)=e^{-\frac{i E t}{\hbar}}\left(\begin{array}{c}
\psi^{(1)}(x, y) \\
\psi^{(2)}(x, y)
\end{array}\right),
$$

then the Dirac equation (83) can be written as a coupled equation system

$$
\begin{aligned}
& -s c \hbar \frac{\partial \psi^{(1)}}{\partial x}-i c \hbar \frac{\partial \psi^{(1)}}{\partial y}+e\left(A_{y}-i s A_{x}\right) \psi^{(1)}-\left(E+m c^{2}+V-e A_{t}\right) \psi^{(2)}=0 \\
& -s c \hbar \frac{\partial \psi^{(2)}}{\partial x}+i c \hbar \frac{\partial \psi^{(2)}}{\partial y}-e\left(A_{y}+i s A_{x}\right) \psi^{(2)}+\left(E-m c^{2}-V-e A_{t}\right) \psi^{(1)}=0 .
\end{aligned}
$$

With the purpose to study a set of soluble systems using the factorization method, we will restrict the following treatment to the case in which the potentials depend only on one spatial coordinate, that we take as the coordinate $x$. With this restriction, the wavefunctions $\psi^{(1,2)}(x, y)$ can be written in the form

$$
\psi^{(1,2)}(x, y)=e^{\frac{i k y}{\hbar}} \psi_{1,2}(x),
$$

and then the coupled equation system (86) and (87) is written as

$$
\begin{array}{r}
-s c \hbar \frac{d}{d x}\left(\begin{array}{l}
\psi_{1}(x) \\
\psi_{2}(x)
\end{array}\right)+\left(\begin{array}{rr}
-i e s A_{x}(x)+e A_{y}(x) & e A_{t}(x)-V(x) \\
-e A_{t}(x)-V(x) & -i e s A_{x}(x)-e A_{y}(x)
\end{array}\right)\left(\begin{array}{c}
\psi_{1}(x) \\
\psi_{2}(x)
\end{array}\right) \\
=\left(\begin{array}{cc}
-c k & E+m c^{2} \\
-E+m c^{2} & c k
\end{array}\right)\left(\begin{array}{l}
\psi_{1}(x) \\
\psi_{2}(x)
\end{array}\right) .
\end{array}
$$

We can observe that the equation system (89) has the same form as the equation system $(10)$ of the $(1+1)$-dimensional case, in such a way that the potentials $V(x), e A_{y}(x), A_{x}(x)$ and $A_{t}(x)$ of the $(2+1)$-dimensional case play the same role respectively as the potentials $P(x),-V(x),-A_{x}(x)$ and $-A_{t}(x)$ of the $(1+1)$-dimensional case. In general the $A_{x}$ component can be removed by a gauge transformation, without loss of generality, we impose $A_{x}=0$ in the following treatment. 


\subsection{Mix of scalar, electric scalar and vectorial potentials}

The mix of vectorial, scalar and electric scalar potentials depending on the $x$-variable for the $(2+1)$ dimensional case is analogous to the one discussed in section $(2.2)$ for the $(1+1)$ dimensional case. As it was performed in the $(1+1)$ dimensional case, here we also impose the same functional form $g(x)$ for all the potentials which are present in the problem. In this form, we write $e A_{y}(x)=\lambda_{1} g(x), V(x)=\lambda_{2} g(x)$ and $e A_{t}(x)=\lambda_{3} g(x)$, where $\lambda_{1}, \lambda_{2}$ and $\lambda_{3}$ are constants. Following a similar procedure as the one performed for the $(1+1)$-dimensional case, in section $(2.2)$, we obtain that

$$
\begin{aligned}
& -c s \hbar \frac{d}{d x}\left(\begin{array}{c}
\tilde{\psi}_{1}(x) \\
\tilde{\psi}_{2}(x)
\end{array}\right)+e A_{y}(x)\left(\begin{array}{cc}
\tau & 0 \\
0 & -\tau
\end{array}\right)\left(\begin{array}{c}
\tilde{\psi}_{1}(x) \\
\tilde{\psi}_{2}(x)
\end{array}\right) \\
& =\left(\begin{array}{cc}
-\frac{E_{n} \nu+\lambda m c^{2}+c k}{\tau} \\
\frac{\lambda\left(m c^{2}-c k \lambda\right)+E_{n} \nu+c k \nu^{2}-\left(E \lambda+m c^{2} \nu\right) \tau}{\tau(1-\nu)}
\end{array}\right)\left(\begin{array}{c}
\tilde{\psi}_{1}(x) \\
\tilde{\psi}_{2}(x)
\end{array}\right),
\end{aligned}
$$

where $\lambda=\lambda_{2} / \lambda_{1}, \nu=\lambda_{3} / \lambda_{1}$ and $\tau=\sqrt{1+\lambda^{2}-\nu^{2}}$. For this case, the ladder operators are

$$
\begin{aligned}
A & =\operatorname{sch} \frac{d}{d x}-\tau e A_{y}(x)-\frac{E \nu+\lambda m c^{2}+c k}{\tau}, \\
A^{\dagger} & =-\operatorname{sch} \frac{d}{d x}-\tau e A_{y}(x)-\frac{E \nu+\lambda m c^{2}+c k}{\tau} .
\end{aligned}
$$

If the condition $1+\lambda^{2}>\nu^{2}$ is satisfied, the symmetric partner Hamiltonians are energy dependent and the operators $A$ and $A^{\dagger}$ are mutually self-adjoint. For the other case, $\tau$ is purely imaginary and the operators $A$ and $A^{\dagger}$ are no longer mutually self-adjoint. The supersymmetric partners for this case are

$$
\begin{aligned}
& H_{-} \tilde{\psi}_{1}(x)=A^{\dagger} A \tilde{\psi}_{1}(x)=\varpi \tilde{\psi}_{1}(x), \\
& H_{+} \tilde{\psi}_{2}(x)=A A^{\dagger} \tilde{\psi}_{2}(x)=\varpi \tilde{\psi}_{2}(x),
\end{aligned}
$$

where

$\varpi=\left(\frac{E^{2}\left(1+\lambda^{2}\right)-\left(m c^{2}-c k \lambda\right)^{2}+2 E\left(c k+\lambda m c^{2}\right) \nu+\left(c^{2} k^{2}+m^{2} c^{4}\right) \nu^{2}}{\tau^{2}}\right)$.

4.1.1. Mix of a scalar linear potential and a constant electromagnetic field

Now we will solve the Dirac equation in the presence of a scalar linear potential $V=C x$, a magnetic field perpendicular to the $x y$-plane with a 
negative direction over the $z$ axis $e A_{y}(x)=-e \mathbb{B} x$ and a constant electric field in the $x$ direction $A_{t}=-\mathbb{E} x$. For this case $\lambda=-C / e \mathbb{B}, \nu=\mathbb{E} / \mathbb{B}$ and $\tau=\sqrt{1+(C / e \mathbb{B})^{2}-(\mathbb{E} / \mathbb{B})^{2}}$ and the ladder operators for the case $s=1$ are defined as

$$
\begin{gathered}
A=\frac{d}{d x}+\tau e \mathbb{B} x-\frac{E \nu+\lambda m c^{2}+c k}{\tau}, \\
A^{\dagger}=-\frac{d}{d x}+\tau e \mathbb{B} x-\frac{E \nu+\lambda m c^{2}+c k}{\tau} .
\end{gathered}
$$

The definition of the ladder operators changes for the case $s=-1$, in such a way that $A \leftrightarrow A^{\dagger}$. The problem that we are considering here is completely analogue to the one studied in the $(2+1)$-dimensional case, which was presented in section (3.2.1). If we follow the same procedure as the one developed in the $(1+1)$-dimensional case, the eigenvalues are obtained from the solution of Eq. (95). These eigenvalues are given by

$$
E_{n}=\frac{-\nu\left(\lambda m c^{2}+c k\right) \pm \sqrt{\tau^{2}\left(\left(c k \lambda-m c^{2}\right)^{2}+2 \tau|e \mathbb{B}| c \hbar n\left(1+\lambda^{2}\right)\right)}}{1+\lambda^{2}}
$$

For the case in which $m=\lambda=0$, the energy spectrum that we obtain is the same as the one found by Lukose et al. [42]. These authors have used the fact that if $\mathbb{B}>\mathbb{E}$, then it is possible always to boost to a frame of reference where the electric field vanishes and the magnetic field is reduced. In this form, these authors have obtained the spectrum associated to this new magnetic field and finally they apply the inverse boost transformation to obtain the spectrum [42]. We note that the range of validity of the procedure developed by these authors is equivalent to the one developed here $(\mathbb{B}>\mathbb{E})$. Similar

conditions for the existence of bound states for the $(3+1)$-dimensional KleinGordon and Dirac equations in the presence of a mix between electric and magnetic fields were found by Adame et al. [10]. Other possible application of the method presented in this work, related to the most general combination of potentials having the same functional form, is the obtention of the spectrum for the system defined by the $(2+1)$-dimensional Dirac oscillator in the presence of an external uniform magnetic field [43].

\section{Supersymmetric partners and Klein paradox}

The $(1+1)$-dimensional Schrödinger equation in the presence of a linear scalar potential of the form $V(x)=l+k|x|$ has been satisfactorily solved 
and leads to the existence of bound states [44]. However, for the $(1+1)$ dimensional Dirac equation in the presence of a linear electric scalar potential of the form $A_{t}=k x$, it has been found that there is no bound state solution $[45,46]$. A simple form to obtain this result is to set the gauge potential as $A_{\mu}=\left(A_{t}(x), 0\right)=(k x, 0)$ in the Eq. (1). For this case, we can obtain from (10) the following decoupled equation system

$$
\begin{aligned}
H_{\mp} \psi_{1(2)}(x) & =\left[-c^{2} \hbar^{2} \frac{d^{2}}{d x^{2}}-e^{2} k^{2} x^{2}-2 e k x E-E^{2} \pm i e \hbar k\right] \psi_{1(2)}(x) \\
& =-m^{2} c^{4} \psi_{1(2)}(x),
\end{aligned}
$$

where $H_{\mp}$ are not supersymmetric partners because these Hamiltonians cannot be constructed in terms of the two operators $A$ and $A^{\dagger}$ mutually selfadjoints. We observe that in Eq. (99) the term $e^{2} k^{2} x^{2}$ is dominant and the asymptotic behavior of the eigenfunctions are determined by the equation

$$
\left[c^{2} \hbar^{2} \frac{d^{2}}{d x^{2}}+e^{2} k^{2} x^{2}\right] \psi_{1(2)}(|x| \rightarrow \infty)=0 .
$$

The behavior of Eq. (100) has been very well studied by Saradzhev [46], who has show that the solutions do not belong to $L^{2}$, thus there are not bound states. The absence of bound state solutions in this problem has been attributed to the Klein paradox [15, 45]. Additionally, the problem of a mixed scalar-electric scalar linear potentials in $(1+1)$ dimensions has been studied by Castro [12], who found bound state solutions for the case in which the scalar coupling has the sufficient intensity compared to the electric scalar coupling. The last result is a particular case of the problem that we have considered in the example 3.2.1.

Additionally to the above example, it is possible to find in the literature more examples in which if the intensity of the electric scalar potential is higher than the one of the scalar potential then there are not bound state solutions. For instance, although the $(1+1)$-dimensional Schrödinger equation in the presence of an inverse linear potential has been rightly solved by Ran et al. [47], for the $(1+1)$-dimensional Dirac equation in the presence of an electric scalar inverse linear potential of the form $A_{t}(x)=-k /|x|$, it has been observed the non-existence of bound states solutions [18]. As it was noted previously in the example 3.2.2, for the case in which the coupling intensity of the electric scalar potential does not exceed the coupling intensity of the scalar and pseudoscalar potentials (this means for the case in which 
$\tau$ is real), the $(1+1)$-dimensional Dirac equation in the presence of a mix between scalar, pseudoscalar and electric scalar inverse linear potentials has associated bound state solutions. This result is in agreement with the condition found by Castro, for the case of mixed scalar-electric scalar potentials $[16]$.

The solution of the $(1+1)$-dimensional Dirac equation in the presence of a scalar potential $V(x)$ leads to energy levels $E$ which are symmetric with respect to $E=0$ (see, for instance, references [15, 48, 49]). This symmetric behavior can be understood taking into account that if $\Psi(x, t)$ represents an eigenfunction of Eq. (1), for the case $A_{t}(x)=0$ and $V(x) \neq 0$, having an associated energy level $+E$, it is possible to prove that $\sigma^{3} \Psi^{*}(x, t)$ represents also an eigenfunction of the same equation but having an associated energy level $-E$. However, if now $\Psi(x, t)$ is an eigenfunction of Eq. (1), but for the case $V(x)=0$ and $A_{t}(x) \neq 0$, having an associated energy level $+E$, then $\sigma^{3} \Psi^{*}(x, t)$ is not an eigenfunction of the same equation having an associated energy level $-E$. Therefore, the solution of the Dirac equation in the presence of an electric scalar potential $A_{t}(x)$ leads to energy levels which are not symmetric with respect to $E=0$. This is an example that shows one of the differences between the physics which is described by the Dirac equation in the presence of a scalar potential $V(x)$ and an electric scalar potential $A_{t}(x)$, considering that both potentials have the same functional form.

We have studied in sections 2 and 4 the Dirac equation in $(1+1)$ and $(2+1)$ dimensions in the presence of static potentials having the same functional form. We have found that the Dirac equations (14) and (90) can be looked like Dirac equations in the presence of scalar potentials with rescaled couplings $V(x) \rightarrow \tau V(x)$. This means that if the coupling of the electric scalar potential $A_{t}$ has an intensity for which the parameter $\tau$ is real, then for this case there is no mix between the positive and negative energy states, in the same way as the scalar potential does not. The condition for $\tau$ to be real implies that if we can build two Schrödinger supersymmetric partner Hamiltonians for the Dirac equation, then the Dirac sea is stable and the variations of the couplings in the potentials provides a way to avoid the Klein paradox.

It is worth noting that the condition for $\tau$ is necessary but not sufficient for establishing the absence of the Klein paradox. To illustrate the validity of this sentence, we consider the $(1+1)$-dimensional Dirac equation in the presence of mixed scalar-electric scalar step potential. For this case, the sufficient condition for the spontaneous production of particle-antiparticle 
pairs is $l>1+2 m c^{2} / V$, where $V$ is the scalar potential. In our case, if $\tau$ is not real, means that $l>1$. Therefore, for the interval $1+2 m c^{2} / V>l>1$ there is not the Klein paradox, but our condition cannot predict the fact that the vacuum is stable for this interval. In this sense, this condition is necessary but is not sufficient.

\section{Conclusions}

In this work we have studied the $(1+1)$ and $(2+1)$-dimensional Dirac equations in the presence of static external potentials. We have considered the mix of the potentials as the most general combination of Lorentz structures, for the case in which the potentials have the same functional. After we have diagonalized this problem, we have arrived to rewrite the Dirac equation in terms of two first-order differential operators, which are called ladder operators, and we have showed that the problem has been mapped to a Dirac equation in the presence of a scalar potential with rescaled coupling. If the ladder operators $A$ and $A^{\dagger}$ are mutually self-adjoint, then the Dirac sea is stable, therefore, there is not the Klein paradox and the Dirac particles can be confined. The factorization method has been also generalized for energydependent Hamiltonians and the shape invariance for a restricted class of energy-dependent Hamiltonians.

\section{Acknowledgments}

J. A. Sánchez thanks CAPES for financial support.

\section{References}

[1] R. Gerritsma, G. Kirchmair, F. Zähringer, E. Solano, R. Blatt, and C. F. Roos, Nature (London) 463 (2010) 68.

[2] R. Gerritsma, B. P. Lanyon, G. Kirchmair, F. Zähringer, C. Hempel, J. Casanova, J. J. Garcia-Ripoll, E. Solano, R. Blatt, and C. F. Roos, Phys. Rev. Lett. 106 (2011) 060503.

[3] J. Casanova, J. J. García-Ripoll, R. Gerritsma, C. F. Roos, and E. Solano, Phys. Rev. A 82 (2010) 020101.

[4] N. M. R. Peres, F. Guinea, and A. H. Castro Neto, Phys. Rev. B 73 (2006) 205408. 
[5] M. I. Katsnelson, K. S. Novoselov, and A. K. Geim, Nature Phys. 2 (2006) 620.

[6] V. B. Bagrov and D. M. Gitman, Exact solutions of relativistic wave equations, Kluwer Academic Publishers, 1990.

[7] B. Thaller, The Dirac equation, Springer-Verlag Berlin Heildeberg, 1992.

[8] F. Cooper, A. Khare, and U. Sukhatme, Phys. Rep. 251 (1995) 267.

[9] F. Domínguez-Adame and M. A. González, Europhys. Lett. 13 (3) (1990) 193.

[10] F. Dominguez-Adame and B. Méndez, Nuovo Cimento B 107 (1992) 489.

[11] C. Y. Long and C. Y. Qin, Chin. Phys. 16 (2007) 897.

[12] A. S. de Castro, Phys. Lett. A 305 (2002) 100.

[13] A. S. de Castro and W. G. Pereira, Phys. Lett. A 308 (2003) 131.

[14] A. S. de Castro, Phys. Lett. A 318 (2003) 40.

[15] A. S. de Castro, Phys. Lett. A 328 (2004) 289.

[16] A. S. de Castro, Ann. Phys. 316 (2005) 414.

[17] L. B. Castro, A. S. de Castro and M. Hott, Int. J. Mod. Phys. E, 16 (2007) 3002.

[18] S. H. Dong, J. Phys. A 36 (2003) 4977.

[19] S. H. Dong and Z. Q. Ma, Phys. Lett. A 312 (2003) 78.

[20] S. Zarrinkamar, A. Rajabi, and H. Hassanabadi, Ann. Phys. 325 (2010) 1720.

[21] R. Hughes, V. A. Kostelecky and M. M. Nieto, Phys. Rev. D 34 (1986) 1100.

[22] J. Beckers and N. Debergh, Phys. Rev. D 42 (1990) 1255. 
[23] P. A. M. Dirac, The Principles of Quantum Mechanics (Clarendon Press, Oxford) 1935.

[24] E. Schrodinger, Proc. R. Irish Acad. A 46 (1940) 9; 46 (1940) 183; 47 (1941) 53.

[25] L. Infeld and T. E. Hull, Rev. Mod. Phys. 23(1) (1951) 21-68.

[26] G. Darboux, C. R. Acad, Sci. (Paris) 94 (1882) 1456.

[27] E. Witten, Nucl. Phys. B 188 (1981) 513.

[28] L. Gendenshtein, JETP Lett. 38 (1983) 356.

[29] R. P. Martínez y Romero, M. Moreno, and A. Zentella, Phys. Rev. D 43 (6) (1991) 2036.

[30] J. Benitez, R. P. Martinez y Romero, H. N. Nuñez-Yépez and A. L. Salas-Brito, Phys. Rev. Lett. 64 (1990) 1643.

[31] A. Sinha and P. Roy, Mod Phys. Lett. A, 20 (31) (2005) 2377.

[32] A. Sinha and P. Roy, Int. J. Mod. Phys A, 21 (2006) 5807.

[33] V. G. dos Santos, A. de Souza Dutra, and M. B. Hott, Phys. Lett. A, 373 (38) (2009) 3401.

[34] S. H. Dong, Factorization Method in Quantum Mechanics, Springer, 2007.

[35] B. K. Bagchi, Supersymmetry in quantum and classical mechanics. Chapman and Hall-CRC, 2010.

[36] J. Formánek, R. J. Lombard, and J. Mareš, Czech. J. Phys. 54(3) (2004) 289.

[37] J. García-Martínez, J. García-Ravelo, J. J. Pena, and A. SchulzeHalberg, Phys. Lett. A 1 (2009) 5.

[38] H. Hassanabadi, E. Maghsoodi, R. Oudi, S. Zarrinkamar, H. Rahimov, Eur. Phys. J. Plus 127 (2012) 120. 
[39] R. Yekken, M. Lassaut, and R. J. Lombard, Few-Body Systems (2013) 1.

[40] A. Gangopadhyaya, J. V. Mallow, and C. Rasinariu, Supersymmetric Quantum Mechanics: An Introduction World Scientific, (2010).

[41] J. G. Xing and R. Z. Zhou, Commun. Theor. Phys. 49 (2008) 319.

[42] V. Lukose, R. Shankar, and G. Baskaran, Phys. Rev. Lett. 98 (2007) 116802 .

[43] C. Quimbay and P. Strange, (2013). arXiv:1312.5251.

[44] O. Vallée and M. Soares M, Airy Functions and Applications to Physics, London: Imperial College Press, 2004.

[45] H. Galic, Am. J. Phys. 56 (1988) 312.

[46] F. Saradzhev, J. Phys. A: Math. Gen. 34 (2001) 1771.

[47] Y. Ran, L. Xue, S. Hu, and R. K. Su, J. Phys. A 33 (2000) 9265.

[48] F. A. B. Coutinho abd Y. Nogami, Phys. Lett. A 124 (1987) 211.

[49] F. A. B. Coutinho, Y. Nogami, and F. M. Toyama, Am. J. Phys. 56 (10) (1988) 904. 